\title{
A universal relation in torsion for a mixture of solid and fluid
}

\author{
MUKESH GANDHI *, K.R. RAJAGOPAL ** and A.S. WINEMAN * \\ * Department of Mechanical Engineering and Applied Mechanics, University of Michigan, \\ Ann Arbor, MI 48109, USA \\ ** Department of Mechanical Engineering, University of Pittsburgh, Pittsburgh, PA 15261, USA
}

(Received November 2, 1983)

\begin{abstract}
In his study of combined finite extension and torsion of a nonlinear, incompressible, isotropic elastic circular cylinder, Rivlin [1] established a relation for the torsional stiffness which depends only on the axial force, the axial extension ratio and the radius of the undeformed cylinder, in the case of small twist. The relationship did not depend on the structure of the stored energy function and is hence a "universal relation". In this paper, we extend Rivlin's result to the case of combined extension and torsion of a cylindrical mixture of a nonlinear elastic solid and fluid.
\end{abstract}

\section{Introduction}

In his study of the combined finite extension and torsion of a circular cylinder of a nonlinear elastic, incompressible, isotropic material, Rivlin [1] established a striking result in the case of small twist. He exhibited a relation for the torsional stiffness (twisting moment per angle of twist) which depends only on the axial force, the axial extension ratio and the radius of the undeformed cylinder, and does not depend on the mathematical structure of the stored energy function of the nonlinearly elastic material. This relation has been termed a "Universal Relation" because it is the same for all nonlinearly elastic, incompressible, isotropic materials. In this paper we extend Rivlin's result to the case of combined extension and torsion of a cylindrical mixture of a nonlinearly elastic solid and fluid.

The first treatment of the problem of combined extension and torsion of a rubber cylinder containing fluid appears to be due to Treloar [2]. In his analysis, the cylinder is assumed to be saturated with the fluid. In addition, the problem is not treated within the context of mixture theory. The present work differs from Treloar's [2] in two respects. First, the problem is studied within the context of the theory of interacting continua. Second, there is no restriction on the fluid content of the mixture, the state of the cylinder could range from being completely dry to fully saturated.

In the present problem, both the solid and fluid constituents are at rest. However, the fluid can be nonhomogeneously dispersed throughout the mixture region, which gives rise to concentration gradients. The physical mechanism for the existence of such gradients is provided by the presence of a diffusive body force which each constituent exerts on the other. However, when the twisting is small, it is found that the fluid is 
dispersed uniformly throughout the mixture (cf. Gandhi, Rajagopal and Wineman [3]). A brief review of the notations and basic equations relevant to a mixture of interacting continua is provided in Section 2. The general problem of torsion superposed on finite extension is formulated and discussed within the context of mixture theory in Section 3. The problem of a "small" twist superposed on a finite extension is studied in detail in the final section.

\section{Preliminaries}

In this section, we provide a brief discussion of the basic balance laws and their consequences which is pertinent to mixture theory. A detailed exposition of the same can be found in Bowen [4] and Atkin and Craine [5].

A mixture of two continua, a solid $S_{1}$ and a fluid $S_{2}$, which are in motion relative to one another is considered. Let

$$
x=\chi_{1}(X, t) \text { and } y=\chi_{2}(Y, t)
$$

denote the motion of the solid and fluid, respectively. Also, let $\boldsymbol{u}, f$ and $\boldsymbol{v}, \boldsymbol{g}$ denote the velocity and acceleration vectors of $S_{1}$ and $S_{2}$, respectively.

The deformation gradient tensor for the solid $S_{1}$ is given by

$$
F=\frac{\partial \chi_{1}}{\partial X}
$$

Let $\rho_{1}$ and $\rho_{2}$ denote the densities of $S_{1}$ and $S_{2}$ at time $t$, measured per unit volume of the mixture. The mean velocity of the mixture and the total density of the mixture are then defined by

$$
\rho w=\rho_{1} u+\rho_{2} v, \quad \rho=\rho_{1}+\rho_{2} .
$$

The appropriate form for the balance of mass for the solid and fluid are

$$
\begin{aligned}
& \rho_{1} \operatorname{det} \boldsymbol{F}=\rho_{10}, \\
& \frac{\partial \rho_{2}}{\partial t}+\operatorname{div}\left(\rho_{2} v\right)=0,
\end{aligned}
$$

where $\rho_{10}$ is the mass density of the solid before forming the mixture.

Let $\boldsymbol{a}$ and $\pi$ denote the partial stress tensors for $S_{1}$ and $S_{2}$, respectively. Let $b$ denote the diffusive body force. In the absence of external body forces, the equations of motion for $S_{1}$ and $S_{2}$ are

$$
\begin{aligned}
& \operatorname{div} \boldsymbol{\sigma}-\boldsymbol{b}=\rho_{1} f, \\
& \operatorname{div} \pi+b=\rho_{2} g,
\end{aligned}
$$

and

$$
\sigma+\pi=\sigma^{T}+\pi^{T} .
$$

Finally, we discuss briefly the constraint which is introduced on the motion of the mixture in virtue of the assumption that both the solid $S_{1}$ and the fluid $S_{2}$ are incompressible in their unmixed reference configuration. If the solid $S_{1}$ and the fluid $S_{2}$ have constant densities $\rho_{10}$ and $\rho_{20}$ and volume $V_{1}$ and $V_{2}$, respectively, in their 
reference configuration and if one further assumes that the volume of the mixture is always constrained to be $V_{1}+V_{2}$, then (cf. Mills [6]), it can be shown that

$$
\frac{\rho_{1}}{\rho_{10}}+\frac{\rho_{2}}{\rho_{20}}=1 \text {. }
$$

We shall assume that the mixture under consideration obeys the constraint expressed by (9).

\section{Problem}

Let us consider a solid circular cylinder whose dimensions in the reference configuration are given by a radius $R_{0}$ and a length $L_{0}$. We shall denote the co-ordinates of a material particle in the reference configuration in a cylindrical co-ordinate system by $(R, \Theta, Z)$. We shall assume that the cylinder is subject to the following deformation

$$
\begin{aligned}
& r=r(R), \\
& \theta=\Theta+\psi \lambda Z,
\end{aligned}
$$

and

$$
z=\lambda Z
$$

where $(r, \theta, z)$ denote the co-ordinates of the particle at $(R, \Theta, Z)$ at the current instant, $\lambda$ and $\psi$ being constants. The above deformation corresponds to a finite elongation (with an associated stretch ratio $\lambda$ ) along the $z$-co-ordinate direction, followed by a rotation of $\psi$ per unit current length.

The Cauchy-Green strain tensor $\boldsymbol{B}$ which is defined as

$$
\boldsymbol{B}=\boldsymbol{F} \boldsymbol{F}^{T}
$$

takes the following form for the above deformation:

$$
\begin{aligned}
\boldsymbol{B} & =\left(\begin{array}{ccc}
\left(\frac{\mathrm{d} r}{\mathrm{~d} R}\right)^{2} & 0 & 0 \\
0 & \left(\frac{r}{R}\right)^{2}+(\psi \lambda r)^{2} & \psi \lambda^{2} r \\
0 & \psi \lambda^{2} r & \lambda^{2}
\end{array}\right) \\
& =\left(\begin{array}{ccc}
\lambda_{r}^{2} & 0 & 0 \\
0 & \lambda_{\theta}^{2}+\left(\psi R \lambda \lambda_{\theta}\right)^{2} & \psi \lambda^{2} \lambda_{\theta} R \\
0 & \psi \lambda^{2} \lambda_{\theta} R & \lambda^{2}
\end{array}\right)
\end{aligned}
$$

where $\lambda_{r}=\mathrm{d} r / \mathrm{d} R$ and $\lambda_{\theta}=r / R$ denote the stretch ratios in the $r$ and $\theta$ directions, respectively. The principal invariants of $B$ are then given as

$$
\begin{aligned}
& I_{1}=\lambda_{r}^{2}+\lambda_{\theta}^{2}\left(1+\psi^{2} R^{2} \lambda^{2}\right)+\lambda^{2}, \\
& I_{2}=\lambda^{2}\left(\lambda_{r}^{2}+\lambda_{\theta}^{2}\right)+\lambda_{\theta}^{2} \lambda_{r}^{2}\left(1+\psi^{2} R^{2} \lambda^{2}\right), \\
& I_{3}=\lambda_{r}^{2} \lambda_{\theta}^{2} \lambda^{2} .
\end{aligned}
$$


We shall find it useful to express the balance of mass equation (for the solid constituent) in terms of the stretch ratios. Thus (4) can be expressed as

$$
\frac{\rho_{1}}{\rho_{10}}=\frac{1}{\lambda_{r} \lambda_{\theta} \lambda}=v_{s},
$$

where $v_{s}$ represents the volume fraction of the solid.

We now proceed to document the equations of equilibrium which are appropriate for the deformation being considered. Since the assumed form of deformation implies that the stresses depend only on the $r$ co-ordinate, the equations of equilibrium for the solid constituent, namely (4), reduces to

$$
\frac{\mathrm{d} \sigma_{r r}}{\mathrm{~d} r}+\frac{\sigma_{r r}-\sigma_{\theta \theta}}{r}-b_{r}=0,
$$

where $\sigma_{r r}$ and $\sigma_{\theta \theta}$ denote the appropriate components of $\sigma$ and $b_{r}$ the component of the diffusive body force $\boldsymbol{b}$ in the radial direction. The equilibrium equation for the fluid constituent, namely (5), reduces to

$$
\frac{\mathrm{d} \pi_{r r}}{\mathrm{~d} r}+\frac{\pi_{r r}-\pi_{\theta \theta}}{r}+b_{r}=0
$$

where $\pi_{r r}$ and $\pi_{\theta \theta}$ denote the components of $\pi$. If $\boldsymbol{T}$ denotes the total stress tensor, i.e.,

$$
\boldsymbol{T}=\boldsymbol{\sigma}+\boldsymbol{\pi}
$$

then (18) and (19) imply that

$$
\frac{\mathrm{d} T_{r r}}{\mathrm{~d} r}+\frac{T_{r r}-T_{\theta \theta}}{r}=0,
$$

which is the equation of equilibrium for the mixture.

We shall assume that the solid-fluid mixture is such that its specific Helmholtz free energy function has the following constitutive structure (cf. [7])

$$
A=\tilde{A}\left(\boldsymbol{F}, \rho_{2}\right) \text {. }
$$

Under the assumption of isotropy and material frame indifference, the free energy function $A$ can be written in terms of an integrity basis for $\boldsymbol{B}$ as

$$
A=\bar{A}\left(I_{1}, I_{2}, I_{3}, \rho_{2}\right) \text {. }
$$

It follows from equations (4), (9) (16) that

$$
\left(I_{3}\right)^{-1 / 2}=1-\frac{\rho_{2}}{\rho_{20}},
$$

and thus $A$ reduces to

$$
A=A\left(I_{1}, I_{2}, \rho_{, 2}\right) \text {. }
$$

The above constitutive assumption seems to be an appropriate one for a mixture of a nonlinearly elastic material like rubber in a solvent (cf. Treloar [8]). The restriction imposed by thermodynamics and the reductions which can be achieved for the forms of the constitutive relations for the stresses have been studied in detail in [7]. We provide below the results obtained therein.

The constitutive relation involving the partial stress components for the solid and 
fluid constituents are given, respectively, by

$$
\sigma_{i j}=\left(\phi-p \frac{\rho_{1}}{\rho_{10}}\right) \delta_{i j}+2 \rho\left\{\left(A_{1}+A_{2} I_{1}\right) B_{i j}-A_{2} B_{i k} B_{k j}\right\}
$$

and

$$
\pi_{i j}=\left(-\phi-p \frac{\rho_{2}}{\rho_{20}}-\rho \rho_{2} \frac{\partial A}{\partial \rho_{2}}\right) \delta_{i j}
$$

The constitutive relation for the components of the diffusive body force is given by

$$
b_{i}=\frac{\partial \phi}{\partial x_{i}}-\frac{p}{\rho_{10}} \frac{\partial \rho_{1}}{\partial x_{i}}+\rho \frac{\partial A}{\partial \rho_{2}} \frac{\partial \rho_{2}}{\partial x_{i}}-\rho_{2}\left\{\left(A_{1}+A_{2} I_{1}\right) \delta_{l k}-A_{2} B_{l k}\right\} \frac{\partial B_{l k}}{\partial x_{i}},
$$

where $\delta_{i j}$ denotes the Kronecker delta and $A_{i}, i=1,2$ is defined through

$$
A_{i}=\frac{\partial A}{\partial I_{i}}, \quad i=1,2
$$

The scalar $p$ is due to the constraint of volume additivity. The scalar $\phi$ was introduced into the theory by Green and Naghdi [9] for thermodynamic considerations. As can be seen from equations (6), (7) and (8) $\phi$ drops out of the field equations. It is only of interest if partial stresses are to be calculated. Since this is not of interest in the present problem, we drop reference to it without loss of generality. Finally, the constitutive relation for the total stress takes the form

$$
\begin{aligned}
T_{i j} & =\pi_{i j}+\sigma_{i j} \\
& =\left(-p+\rho \rho_{2} \frac{\partial A}{\partial \rho_{2}}\right) \delta_{i j}+2 \rho\left\{\left(A_{1}+A_{2} I_{1}\right) B_{i j}-A_{2} B_{i k} B_{k j}\right\} .
\end{aligned}
$$

For the deformation under consideration, it follows from (13) and equations (25)-(27) that

$$
\begin{aligned}
\sigma_{r r}= & \phi-p \frac{\rho_{1}}{\rho_{10}}+2 \rho\left(A_{1}+A_{2} I_{1}\right) \lambda_{r}^{2}-2 \rho\left(A_{2}\right) \lambda_{r}^{4}, \\
\sigma_{\theta \theta}= & \phi-p \frac{\rho_{1}}{\rho_{10}}+2 \rho\left(A_{1}+A_{2} I_{1}\right) \lambda_{\theta}^{2}\left(1+\psi^{2} R^{2} \lambda^{2}\right) \\
& -2 \rho A_{2}\left\{\lambda_{\theta}^{4}\left(1+\psi^{2} R^{2} \lambda^{2}\right)^{2}+\psi^{2} R^{2} \lambda^{4} \lambda_{\theta}^{4}\right\}, \\
\sigma_{z z}= & \phi-p \frac{\rho_{1}}{\rho_{10}}+2 \rho\left(A_{1}+A_{2} I_{1}\right) \lambda^{2}-2 \rho A_{2}\left\{\lambda^{4}\left(1+\psi^{2} R^{2} \lambda_{\theta}^{2}\right)\right\}, \\
\sigma_{\theta z}= & 2 \rho\left(A_{1}+A_{2} I_{1}\right) \psi R \lambda_{\theta} \lambda^{2}-2 \rho A_{2}\left\{\psi R \lambda_{\theta} \lambda^{2}\left[\left(1+\psi^{2} R^{2} \lambda^{2}\right) \lambda_{\theta}^{2}+\lambda^{2}\right]\right\} \\
= & 2 \rho \psi R\left\{\left(A_{1}+A_{2} I_{1}\right) \lambda^{2} \lambda_{\theta}-A_{2}\left[\lambda^{2} \lambda_{\theta}\left(\left(1+\psi^{2} R^{2} \lambda^{2}\right) \lambda_{\theta}^{2}+\lambda^{2}\right)\right]\right\}, \\
\pi_{r r}= & \pi_{\theta \theta}=\pi_{z z}=-p \frac{\rho_{2}}{\rho_{20}}+\rho \rho_{2} \frac{\partial A}{\partial \rho_{2}} .
\end{aligned}
$$

The expression for the diffusive body force is quite complicated and for the purposes of our analysis here it is sufficient to realize that the diffusive body force takes the 
following form:

$$
b_{r}=-p \frac{\mathrm{d}}{\mathrm{d} r}\left(\rho_{1} / \rho_{10}\right)+g\left(\lambda_{r}, \lambda_{\theta}, R, \lambda_{r}^{\prime}, \lambda_{\theta}^{\prime}, \lambda, \psi^{2}\right),
$$

where the prime denotes differentiation with respect to the variable $R$.

It follows from equations (14)-(16) and (28)-(31) that the components of the stress $\sigma$ have the following forms:

$$
\sigma_{i i}=-\frac{\rho_{1}}{\rho_{10}} p+f_{i i}\left(\lambda_{r}, \lambda_{\theta}, \lambda, \psi^{2} R^{2}\right), \text { no sum on } i,
$$

and

$$
\sigma_{\theta z}=\psi R f_{\theta z}\left(\lambda_{r}, \lambda_{\theta}, \psi^{2} R^{2}\right) .
$$

Also, the components of the total stress for the mixture $T$ have the following forms:

$$
T_{i i}=-p+h_{i i}\left(\lambda_{r}, \lambda_{\theta}, \lambda, \psi^{2} R^{2}\right) \text {, no sum on } i,
$$

and

$$
T_{\theta z}=\psi R h_{\theta z}\left(\lambda_{r}, \lambda_{\theta}, \lambda, \psi^{2} R^{2}\right) .
$$

The equilibrium equation for the solid (18) can then be re-written in the form

$$
\begin{aligned}
& \frac{\mathrm{d}}{\mathrm{d} r}\left\{-\frac{\rho_{1}}{\rho_{10}} p+f_{r r}\right\}+\frac{\left(f_{r r}-f_{\theta \theta}\right)}{(r / R)} R+p \frac{\mathrm{d}}{\mathrm{d} r}\left(\frac{\rho_{1}}{\rho_{10}}\right) \\
& \quad+g\left(\lambda_{r}, \lambda_{\theta}, R, \lambda_{r}^{\prime}, \lambda_{\theta}^{\prime}, \lambda, \psi^{2} R^{2}\right)=0 .
\end{aligned}
$$

Thus

$$
-\frac{\mathrm{d} p}{\mathrm{~d} R} \frac{\rho_{1}}{\rho_{10}}+g_{1}\left(\lambda_{r}, \lambda_{\theta}, R, \lambda_{r}^{\prime}, \lambda_{\theta}^{\prime}, \lambda, \psi^{2} R^{2}\right)=0 .
$$

The equation governing the equilibrium of the mixture takes the form

$$
\frac{\mathrm{d} p}{\mathrm{~d} r}=\frac{\mathrm{d} h_{r r}}{\mathrm{~d} r}+\frac{h_{r r}-h_{\theta \theta}}{r},
$$

which is of the form

$$
\frac{\mathrm{d} p}{\mathrm{~d} R}+g_{2}\left(\lambda_{r}, \lambda_{\theta}, R, \lambda_{r}^{\prime}, \lambda_{\theta}^{\prime}, \lambda, \psi^{2} R^{2}\right)=0 .
$$

Equations (39) and (41) are two highly nonlinear second order ordinary differential equations for $r(R)$ and $p(R)$. One boundary condition arises from the assumption that the lateral surface of the deformed cylinder is traction free. This requires that the radial component of the total stress vanishes, i.e. $T_{r r}\left(R_{0}\right)=0$. The choice of a second boundary condition is unclear. For example, there is almost no physical guidance for specifying boundary conditions on the partial stress components. However, for the purposes of this work, which is the determination of a "universal relation", the boundary condition that $T_{r r}\left(R_{0}\right)=0$ is adequate.

Once $r(R)$ and $p(R)$ have been found, the partial and total stress components can be determined. Thus, the twisting moment $M_{z}$ and the axial force $F$ are given by the formulae

$$
M_{z}=2 \pi \int_{0}^{r_{0}} r^{2} T_{\theta z} \mathrm{~d} r=2 \pi \psi \int_{0}^{r_{0}} R r^{2} h_{\theta z} \mathrm{~d} r
$$


and

$$
F=2 \pi \int_{0}^{r_{0}} T z_{z} r \mathrm{~d} r=2 \pi \int_{0}^{r_{0}} r\left(-p+h_{z z}\right) \mathrm{d} r,
$$

where $r_{0}$ is the outer radius of the cylinder in the deformed swollen state.

Since the extent of swelling is not known and thus $r_{0}$ is not known a priori, it would be convenient to express the moment $M_{z}$ and the axial force $F$ in terms of integrals over the known reference configuration. Thus, we can express (42) and (43) as

$$
M_{z}=2 \pi \psi \int_{0}^{R_{0}} \lambda_{r} \lambda_{\theta}^{2} h_{\theta z} R^{3} \mathrm{~d} R
$$

and

$$
F=2 \pi \int_{0}^{R_{0}} \lambda_{r} \lambda_{\theta}\left(-p+h_{z z}\right) R \mathrm{~d} R
$$

\section{Small twist superposed on finite extension}

In this section we consider the case when a small twist is superposed on a finite extension. Thus we shall assume that $\psi \ll 1$ and that we can expand the relevant quantities in a Taylor series expansion in $\psi$. Note that in equations (39) and (41), $g_{1}$ and $g_{2}$ depend on $\psi^{2}$. The solutions to (39) and (41) will be of the forms

$$
r=r\left(R, \psi^{2}\right), \quad p=p\left(R, \psi^{2}\right) \text {. }
$$

Thus we can express $r$ and $p$ by their Taylor series expansions as

$$
\begin{aligned}
& r=\hat{r}(R)+\mathrm{O}\left(\psi^{2}\right), \\
& p=\hat{p}(R)+\mathrm{O}\left(\psi^{2}\right) .
\end{aligned}
$$

Similarly one can expand $\lambda_{r}$ and $\lambda_{\theta}$ in terms of Taylor series expansions in $\psi^{2}$ and the Cauchy-Green strain tensor (13) now takes on the form

$$
\boldsymbol{B}=\left(\begin{array}{lll}
\hat{\lambda}_{r}^{2} & 0 & 0 \\
0 & \hat{\lambda}_{\theta}^{2} & \psi \lambda^{2} \hat{\lambda}_{\theta} R \\
0 & \psi \lambda^{2} \hat{\lambda}_{\theta} R & \lambda^{2}
\end{array}\right)+\mathrm{O}\left(\psi^{2}\right) \equiv \hat{\boldsymbol{B}}+\mathrm{O}\left(\psi^{2}\right)
$$

where

$$
\hat{\lambda}_{r}=\lambda_{r}(R, 0)
$$

and

$$
\hat{\lambda}_{\theta}=\lambda_{\theta}(R, 0) \text {. }
$$

Likewise, the principal invariants $I_{1}, I_{2}$ and $I_{3}$ and the densities $\rho_{1}, \rho_{2}$ and $\rho$ can be expanded to be

$$
\begin{aligned}
& I_{1}=\lambda_{r}^{2}+\lambda_{\theta}^{2}+\lambda^{2}=\hat{\lambda}_{r}^{2}+\hat{\lambda}_{\theta}^{2}+\lambda^{2}+\mathrm{O}\left(\psi^{2}\right) \equiv \hat{I}_{1}+\mathrm{O}\left(\psi^{2}\right), \\
& I_{2}=\lambda_{r}^{2} \lambda_{\theta}^{2}+\lambda^{2} \lambda_{r}^{2}+\lambda^{2} \lambda_{\theta}^{2}=\hat{\lambda}_{r}^{2} \hat{\lambda}_{\theta}^{2}+\lambda^{2} \hat{\lambda}_{r}^{2}+\lambda^{2} \hat{\lambda}_{\theta}^{2}+\mathrm{O}\left(\psi^{2}\right)
\end{aligned}
$$




$$
\begin{aligned}
& =\hat{I}_{2}+\mathrm{O}\left(\psi^{2}\right), \\
I_{3} & =\lambda_{r}^{2} \lambda_{\theta}^{2} \lambda^{2}=\hat{\lambda}_{r}^{2} \hat{\lambda}_{\theta}^{2} \lambda^{2}+\mathrm{O}\left(\psi^{2}\right)+\hat{I}_{3}+\mathrm{O}\left(\psi^{2}\right), \\
\rho_{1} & =\hat{\rho}_{1}+\mathrm{O}\left(\psi^{2}\right), \quad \rho_{2}=\hat{\rho}_{2}+\mathrm{O}\left(\psi^{2}\right), \\
\rho & =\hat{\rho}+\mathrm{O}\left(\psi^{2}\right),
\end{aligned}
$$

with

$$
\begin{aligned}
& \frac{\hat{\rho}_{1}}{\rho_{10}}=\frac{1}{\hat{\lambda}_{r} \hat{\lambda}_{\theta} \lambda}, \quad \frac{\hat{\rho}_{2}}{\rho_{20}}=\left(1-\frac{\hat{\rho}_{1}}{\rho_{20}}\right), \\
& \hat{\rho}=\hat{\rho}_{1}+\hat{\rho}_{2} .
\end{aligned}
$$

Thus, the normal components of the partial and total stress may now be expressed as

$$
\begin{gathered}
\sigma_{i i}=-\frac{\hat{\rho}_{1}}{\rho_{10}} \hat{p}+\hat{f}_{i i}\left(\hat{\lambda}_{r}, \hat{\lambda}_{\theta}, \lambda\right)+\mathrm{O}\left(\psi^{2}\right), \text { no sun on } i, \\
T_{i i}=-\hat{p}+\hat{h}_{i i}\left(\hat{\lambda}_{r}, \hat{\lambda}_{\theta}, \lambda\right)+\mathrm{O}\left(\psi^{2}\right) \text { no sum on } i .
\end{gathered}
$$

The diffusive body force $b$ may be expressed as

$$
b_{r}=-\hat{p} \frac{\mathrm{d}}{\mathrm{d} R}\left(\frac{\hat{\rho}_{1}}{\rho_{10}}\right) \hat{\lambda}_{r}+\hat{g}\left(\hat{\lambda}_{r}, \hat{\lambda}_{\theta}, R, \hat{\lambda}_{r}^{\prime}, \hat{\lambda}_{\theta}^{\prime}, \lambda\right)+\mathrm{O}\left(\psi^{2}\right) .
$$

The equilibrium equations for the solid and the mixture now take the forms

$$
\begin{aligned}
& -\frac{\mathrm{d} \hat{\rho}}{\mathrm{d} R} \frac{\hat{\rho}_{1}}{\rho_{10}}+\hat{g}_{1}\left(\hat{\lambda}_{r}, \hat{\lambda}_{\theta}, R, \hat{\lambda}_{r}^{\prime}, \hat{\lambda}_{\theta}^{\prime}, \lambda\right)+\mathrm{O}\left(\psi^{2}\right)=0, \\
& \frac{\mathrm{d} \hat{p}}{\mathrm{~d} R}+\hat{g}_{2}\left(\hat{\lambda}_{r}, \hat{\lambda}_{\theta}, R, \hat{\lambda}_{r}^{\prime}, \hat{\lambda}_{\theta}^{\prime}, \lambda\right)+\mathrm{O}\left(\psi^{2}\right)=0 .
\end{aligned}
$$

We now proceed to show that the equations $(58)_{1}$ and $(58)_{2}$ in which terms of $\mathrm{O}\left(\psi^{2}\right)$ are ignored govern the basic finite uniaxial extension problem wherein the solution is homogeneous, i.e.,

$$
\hat{\lambda}_{r}=\text { constant }, \quad \hat{\lambda}_{\theta}=\text { constant. }
$$

Since we shall assume that the material is isotropic

$$
\hat{\lambda}_{r}=\hat{\lambda}_{\theta}=\text { constant. }
$$

It follows from (54) that the densities $\hat{\rho}_{1}, \hat{\rho}_{2}$ and $\hat{\rho}$ are constant. Moreover, it follows from the definitions of $\hat{f}_{i i}$ and $\hat{h}_{i i}$ that

$$
\begin{aligned}
& \hat{f}_{i i}=\text { constant }, \\
& \hat{h}_{i i}=\text { constant },
\end{aligned}
$$

and

$$
\hat{f}_{r r}=\hat{f}_{\theta \theta} \text { and } \hat{h}_{r r}=\hat{h}_{\theta \theta} .
$$

The diffusive body force $b$ vanishes since the gradients of the densities and strain tensor components in (25) now vanish. It follows from equations (38), (40), (58) ${ }_{1}$ and $(58)_{2}$ and the definitions of the functions $g_{1}$ and $g_{2}$, that

$$
\begin{aligned}
& \hat{g}_{1}=0, \\
& \hat{g}_{2}=0,
\end{aligned}
$$


and

$$
\frac{\mathrm{d} \hat{p}}{\mathrm{~d} R}=0
$$

It then follows from (36), (60) and the boundary condition on the lateral surface, $T_{r r}=0$, that

$$
\hat{h}_{r r}\left(\hat{\lambda}_{r}, \hat{\lambda}_{\theta}, \lambda\right)=\hat{p}(R)=\text { constant }
$$

Finally, from (13) and (59), the deformation is found to be

$$
r=\hat{r}(R)=\hat{\lambda} R,
$$

where

$$
\hat{\lambda}=\hat{\lambda}_{r}=\hat{\lambda}_{\theta}
$$

is a constant. In the absence of a second boundary condition, $\hat{\lambda}$ remains arbitrary. Hence, the equations $(58)_{1}$ and $(58)_{2}$ in which terms of $\mathrm{O}\left(\psi^{2}\right)$ are ignored, govern the homogeneous finite uniaxial extension problem. Thus the general problem of combined uniaxial tension followed by small twist decouples into a uniaxial tension problem in which the deformation is homogeneous and a torsion problem in which the twisting is small.

We conclude our analysis by deriving a "universal relation" between the twisting moment and axial force under the assumption of small twist.

The expressions for the twisting moment $M_{z}$ and axial force $F$ in (42) and (43) become

$$
M_{z}=2 \pi \psi \int_{0}^{R_{0}} \hat{\lambda}_{r} \hat{\lambda}_{\theta}^{2} h_{\theta z}\left(\hat{\lambda}_{r}, \hat{\lambda}_{\theta}, \lambda, 0\right) R^{3} \mathrm{~d} R+O\left(\psi^{2}\right)
$$

and

$$
F=2 \pi \int_{0}^{R_{0}} \hat{\lambda}_{r} \hat{\lambda}_{\theta}\left[-\hat{p}(R)+h_{z z}\left(\hat{\lambda}_{r}, \hat{\lambda}_{\theta}, \lambda, 0\right)\right] R \mathrm{~d} R+O\left(\psi^{2}\right) .
$$

It follows from (50)-(52), (61)-(66), and the definitions of $\lambda_{r}, \lambda_{\theta}, h_{\theta z}, h_{z x}$ and $h_{r r}$ that

$$
M_{z}=\left\{\pi R_{0}^{4} \hat{\rho} \lambda^{2} \hat{\lambda}^{4}\left(\hat{A_{1}}+\hat{A_{2}} \hat{\lambda}^{2}\right)\right\} \psi+\mathrm{O}\left(\psi^{2}\right)
$$

and

$$
F=2 \pi R_{0}^{2} \hat{\lambda}^{2} \hat{\rho}\left(\hat{A}_{1}+\hat{A}_{2} \hat{\lambda}^{2}\right)\left(\lambda^{2}-\hat{\lambda}^{2}\right)+\mathrm{O}\left(\psi^{2}\right),
$$

where

$$
\hat{A_{i}}=\hat{A}_{i}\left(\hat{I}_{1}, \hat{I}_{2}, \hat{\rho}_{2}\right), i=1,2 .
$$

By (66) and (67),

$$
\lim _{\psi \rightarrow 0} \frac{M_{z} / \psi}{F}=\frac{R_{0}^{2}}{2} \frac{\lambda^{2} \hat{\lambda}^{2}}{\lambda^{2}-\hat{\lambda}^{2}} .
$$

Equation (68) expresses a relationship between $M_{z}$ and $F$ which is independent of the particular form of the specific Helmholtz free energy $A$ and can hence be called a "universal relation". It is valid for all states in which the elastic solid is swollen with fluid. In the absence of fluid, $\rho_{2}=0$, and thus by (9), (17), and (63), $\lambda \hat{\lambda}^{2}=1$. 
In this case, one obtains from (68) that

$$
\lim _{\psi \rightarrow 0} \frac{M_{z} / \psi}{F}=\frac{R_{0}^{2}}{2}\left[\frac{\lambda^{2}}{\lambda^{3}-1}\right],
$$

which is the classical expression established by Rivlin [1].

We provide below alternate forms for the "universal relation" (68) in terms of other parameters which are of physical interest:

Volume fraction of the solid

$$
\lim _{\psi \rightarrow 0} \frac{M_{z} / \psi}{F}=\frac{R_{0}^{2}}{2} \frac{\lambda^{2}}{\lambda^{3} v_{s}-1}
$$

Solid density

$$
\lim _{\psi \rightarrow 0} \frac{M_{z} / \psi}{F}=\frac{R_{0}^{2}}{2} \frac{\lambda^{2}}{\lambda^{3} \rho_{1} / \rho_{10}-1}
$$

Fluid density

$$
\lim _{\psi \rightarrow 0} \frac{M_{z} / \psi}{F}=\frac{R_{0}^{2}}{2} \frac{\lambda^{2}}{\lambda^{3}\left(1-\rho_{2} / \rho_{20}\right)-1} .
$$

Note that if the cylinder undergoes free expansion when it swells with fluid, isotropy of the material implies $\hat{\lambda}_{R}=\hat{\lambda}_{\theta}=\lambda$. Then $\hat{\lambda}=\lambda$ and the axial force $F=0$ by (67). Thus, the "universal relation" (68) is defined only when $\lambda \neq \hat{\lambda}$, in which case $F \neq 0$.

\section{Acknowledgement}

K.R. Rajagopal would like to acknowledge the solid mechanics program of the National Science Foundation and the Mathematics Research Center at the University of Wisconsin, Madison for their support.

\section{References}

[1] R.S. Rivlin, Large elastic deformations of isotropic materials, VI. Further results in the theory of torsion, shear and flexure. Phil. Trans. Roy. Soc. London A242 (1949) 173-195.

[2] L.R.G. Treloar, Swelling of a rubber cylinder in torsion: Part 1. Theory. Polymer 13 (1972) 195-202.

[3] M. Gandhi, K.R. Rajagopal and A.S. Wineman, Combined tension-torsion of a cylindrical mixture of an elastic solid and fluid. Mathematics Research Center Technical Summary Report.

[4] R.M. Bowen, Theory of mixtures. In: A.C. Eringen (ed.) Continuum Physics, Vol. 3, Academic Press, New York (1978). 
[5] R.J. Atkin and R.E. Craine, Continuum theories of mixtures: Basic theory and historical development. Quart. J. Mech. Appl. Math. 29(2) (1976) 209-244.

[6] N. Mills, Incompressible mixtures of Newtonian fluids. Int. J. Eng. Sci 4 (1966) 97-112.

[7] J.J. Shi, K.R. Rajagopal and A.S. Wineman, Applications of the theory of interacting continua to the diffusion of a fluid through a nonlinear elastic media. Intl. J. Eng. Sci 19 (1981) 871-889.

[8] L.R.G. Treloar, The Physics of Rubber Elasticity, Oxford (1975).

[9] A.E. Green and P.M. Naghdi, On basic equations for mixtures. Quart. J. Mech. \& Appl. Math. 22 (1969) $427-438$. 